\title{
CALIDAD DE VIDA Y TRASTORNO DE ANSIEDAD GENERALIZADA EN LA POBLACIÓN URBANA, MARGINAL Y RURAL DE HUANCAYO*
}

\author{
Cornejo Báez, Walter ${ }^{1}$ y Pérez Camborda, Belén²
}

Facultad de Pedagogía y Humanidades de la Universidad Nacional del Centro del Perú

\begin{abstract}
RESUMEN
Investigación correlacional que tiene por objetivo analizar la asociación entre calidad de vida y trastorno de ansiedad generalizada. Participaron pobladores jóvenes-adultos, varones y mujeres de tres zonas: urbana, marginal y rural del distrito de Huancayo. Para la recopilación de datos se utilizó la Escala de calidad de vida (ECV) de Olson \& Barnes y el Cuestionario de preocupación Pensylvania State de Meyer, Meteggar y Berkovec (TAG) que se aplicó de manera individual a una muestra de 364 sujetos. Los datos obtenidos se procesaron por la prueba chi cuadrada al 0,05 y 0,01 de significación estadística que permitió verificar la prevalencia de baja calidad de vida y tanto la ausencia como la presencia relativa de trastornos de ansiedad generalizada entre los pobladores de Huancayo; las variables sociodemográficas: género y tipo de zona, no tiene mayor incidencia como elementos que diferencien.
\end{abstract}

Palabras clave: calidad de vida, trastorno de ansiedad generalizada, género.

\section{QUALITY OF LIFE AND DYSFUNCTION OF ANXIETY WIDESPREAD IN THE URBAN -MARGINAL AND RURAL POPULATION FROM HUANCAYO}

\begin{abstract}
The correlational investigation that has as a main goal to analyze the relationship between quality of life and dysfunction of anxiety widespread in the young - adults residents both men and women who participate: in this studding coming from an urban, urban marginal and rural district from Huancayo. For the compilation of data the scale of quality of life from Olson \& Barnes (SOL) and a questionaire of comers from pensylvania state of Meyer, Meteggar and Bercovec (TAG) that was applied individually to a sample of 364 fellows. The obtained data were processed by the test chi square at 0,05 and 0,01 of statistical significance which permitted to verify the prevalence of low quality of life and both the absence as the relative presence of anxiety widespread among the residents from Huancayo; the socio - demográphic: gender and area type, don't have bigger incidence like elements that differ each other.
\end{abstract}

Key words: quality of life, dysfunction of anxiety widespread, gender.

* Este trabajo de investigación fue recibido el 20/04/2007, retornado para su revisión el 22/09/2008 y aprobado para su publicación el 27/02/2009.

1. E-mail:wcornejobaez@yahoo.es

2. E-mail: bechoperez@hotmail.com 


\section{INTRODUCCIÓN}

En la sociedad actual se advierte una serie de problemas como: el acelerado crecimiento urbano desordenado, la falta de trabajo o desempleo, la competitividad, el consumismo y el deterioro físico del medio ambiente (Blanco, 1991). Estos hechos traen como consecuencias: modificaciones del estilo de vida, trastornos psicosomáticos; enfermedades: cáncer, trastornos cardiovasculares, enfermedades respiratorias y metabólicas; problemas sociales: alcoholismo, violencia sexual y familiar, pobreza, etc. conducta antisocial, estrés ambiental (Blanco, 1991), aburrimiento, ansiedad, irritación, problemas alimenticios (Carpio, 2000), así como conductas ecológicas irresponsables y problemas del medio urbano (Zinmermann, M 1998); problemas que repercuten en la calidad de vida y la estabilidad emocional de los habitantes $y$, en este contexto, es posible advertir que la calidad de vida (CV) o el estilo de vida de los pobladores de Huancayo, muestre por los cambios ocurridos en esta última década, (que muchos problemas continúen y otros se agudicen), problemas vinculados con la ansiedad. Algunas costumbres permanecen constantes aun por los cambios, tales como los patrones culturales alimenticios por parte de los campesinos y otros, por los cambios de la modernidad, se plasman en la modificación de los estilos y la calidad de vida.

Un tema, así requiere de un estudio pormenorizado que conceptúe los constructos: calidad de vida y ansiedad (según la nueva nomenclatura (DSM-IV) como trastorno de ansiedad generalizada - TAG) y someter a verificación empírica en el contexto concreto de Huancayo. Merecen, por su novedad y complejidad ser analizados de manera empírica y poder ofrecer información científica desde la perspectiva de la psicología social o de la aplicación de la psicología social en salud y en la calidad de vida. Precisamente el interés por el bienestar de los ciudadanos, concede al fenómeno de la calidad de vida y los desajustes del comportamiento, un significado psicosocial y de salud.

Por las consideraciones señaladas, surge el interés y la necesidad de indagar los siguientes problemas:

¿Qué relación existe entre la calidad de vida y el trastorno de ansiedad generalizada de los pobladores de las zonas urbana, marginal y rural del distrito de Huancayo?

¿Los pobladores de zona rural y urbana mostrarán diferencias en los niveles de calidad de vida y trastorno de ansiedad generalizada según el género y tipo de zona?

Se pretende establecer las siguientes hipótesis: Existiría correlación directamente proporcional entre la calidad de vida y el trastorno de ansiedad generalizada (TAG), así entonces a mayor calidad de vida, habría menor presencia de trastorno de ansiedad generalizada. Por las diferencias en las variables sociodemográficas de la población (urbana, marginal y rural), existirían diferencias en la calidad de vida y el trastorno de ansiedad generalizada, siendo mejor la calidad de vida y puntuaciones menores de TAG en sujetos de zona rural y no así en los de zona urbana.

Las variables dependientes a estudiar son:

a) Calidad de vida, que involucra siete dimensiones: hogar y bienestar económico ( $\mathrm{H}$ y $\mathrm{BE})$; Amigos, vecindario y comunidad ( $\mathrm{A}, \mathrm{V}$ y $C$ ); Vida familiar $\mathrm{y}$ familia extensa (VF y FE); educación y ocio ( $E$ y O); medios de comunicación (MC); religión (R) y salud (S).

b) Trastorno de ansiedad generalizada (TAG) con dos niveles: STAG no clínica y STAG clínica.

c) Variables sociodemográficas: género (varón y mujer), zona rural, zona urbana y zona marginal

Esta investigación pretende: describir y analizar las características de la calidad de vida, el comportamiento saludable y establecer sus relaciones; concienciar el problema de laCV confines devalorar comportamientos saludables y mejorar la calidad de vida de la población en estudio y analizar la asociación de la calidad de vida y el trastorno de ansiedad generalizada en función con las variables sociodemográficas (zona geográfica: urbana, marginal y rural), género (varón y mujer).

La investigación sobre calidad de vida y la presunción de enfermedad o de desajustes del comportamiento, son temas que atañen a la psicología social y de la salud o de manera interdisciplinarios, a la psicología social de la salud. 
El estudio de la salud motivó la aparición de la psicología de la salud, que Rodríguez M. (1995); Lamas H. y Murrugarra. A. (2007) y Oblitas (2005), definen como un campo de aplicación de los conocimientos teórico prácticos de la psicología (en su triple vertiente científica, investigadora y profesional) para lograr los objetivos que el sistema de salud plantea en un contexto social determinado; estudia factores emocionales, cognitivos y comportamentales asociados con la salud y enfermedades físicas de los individuos, y colabora en el diseño y aplicación de programas de intervención individuales, grupales y comunitarios, para la promoción de la salud, la prevención tratamiento y rehabilitación de la enfermedad, y para conseguir una buena CV del enfermo.

La adopción de un concepto integral de salud y el tratamiento científico a través de la psicología de la salud, permitió, el surgimiento y desarrollo de la psicología social de la salud como una nueva disciplina para comprender las variables psicológicas y socioculturales de la enfermedad y la salud, cuyos propósitos se enmarcan en el desarrollo de la personalidad saludable (Marciaaton, D. y Xavier F. 1999) o el de autoeficacia (Moscoso, M. 1998), definida como la creencia de una persona en sus propios recursos y habilidades, los cuales le permiten movilizar un adecuado nivel de motivación para el inicio de un plan de acción destinado a ejercer control sobre su comportamiento.

La autoeficacia es un sentimiento de poder y creencia en la capacidad personal, por eso se convierte en parte integral del comportamiento social proactivo.

De la revisión de trabajos sobre calidad de vida en el ámbito internacional se desprenden los realizados por Guri, Veroff y Feld (1960, cit. por Levi L. y Anderson L., 1990); los de Campbell y Converse (1970); los de (Campbell, Converse y Rogers, 1976) así como los de Levi y Anderson (1975, citados por Romero J., 2001).

Tenemos también los de Pol y Domínguez (1986); el de Pol, Guardia y col, (1991); Adam (2000), Roales-Nieto y col.(2004), Tonon (2004), Maric (2005), entro otros.

De estos estudios se puede colegir, que la mayoría de ellos utilizaron diferentes metodologías e instrumentos de medida que no permiten comparaciones con los resultados que se ofrecen, pero que son referencias del estado actual de la investigación internacional.

En el ámbito nacional, el tema de la calidad de vida tiene poca data. A partir de 1998, Schwartz inició con un estudio psicométrico de validación del índice de calidad de vida en Lima. Bulnes et al (1999) estudiaron la CV y la comunicación entre madres adolescentes de Lima mediante la escala de CV de Olson y Barnes.

Tenemos asimismo, los trabajos de Ramírez (2000); de Díaz y cols (2000), la escala de desórdenes emocionales (API) de Anicama, el de Alarcón (2000b); el de Hernández et al (2001), así como el de Cornejo y Cornejo (2002). Barbarán et al (2003), Grimaldo (2003). Rubina y Rebaza (2005), y una investigación reciente fue realizado por Guerra (2006).

En tanto que los estudios previos sobre el trastorno de ansiedad generalizada no figuran, de manera específica, en la literatura internacional y nacional aun cuando el concepto se difundió en 1995, con la publicación del DSM-IV. Lazo (1984) no registra estudios vinculados con el tema y por su parte, Cornejo (1995) al realizar la evaluación de las tesis de Psicología en Perú (1977 a 1994) no observó investigación alguna sobre los temas de calidad de vida.

En el ámbito nacional. Kellert y cols (1967) de modo concreto en el valle del Mantaro, indican que las mujeres evidencian mayor tensión que los varones, los individuos más jóvenes muestran menos tensión que las personas de mayor edad. Los individuos solteros, denotan menos tensión y los viudos o separados tienen la más alta tensión; el mayor optimismo está expresado en los grupos de baja tensión. Villar (1976) reporta la relación entre ansiedad y consumo de drogas en estudiantes preuniversitarios; Velarde (1977) la ansiedad en relación con el autoconcepto en adolescentes de Lima; Ruiz (1981) la ansiedad y temores en adolescentes de la zona central del país; Inga (1999) en 184 estudiantes, varones y mujeres de la UNFV, utilizando el IDAR de Spielberg y el Inventario de Autoestima de Barskdale identificó una correlación negativa entre autoestima y ansiedad, e informó de diferencias según el género, edades y año de estudios. Por último Rubina \& Rebaza (2005), hallaron que las mujeres son dos veces más vulnerables que los hombres a la inestabilidad, la depresión y la ansiedad, 
no así los varones. Las mujeres presentan una mejor felicidad que los varones cuando se valoran emociones positivas.

\section{Calidad de vida}

La calidad de vida es analizada como concepto subjetivo (Blanco, 1985, Zinmermann, 1998, Cornejo, 2003b) de satisfacción de los pobladores en diferentes dimensiones, educación, salud, economía, vivienda y áreas psicosociales.

El concepto de CV está muy ligado a la definición de salud, dada por la OMS. En la literatura sobre el tema, calidad de vida y bienestar son considerados como sinónimos. Bienestar en la lengua anglosajona se refiere a dos términos Welfare y Well-being. El primero, se refiere al estado de bienestar propio de la literatura sociológica económica y política, y el segundo a un estar-bien de carácter más individual o más psicológico.

La CV se convierte en un tema de importancia en la ciencia psicológica y su concepto es complejo. Se propusieron muchas definiciones. En especial destacan aquellas que acentúan el bienestar físico psicológico grupal y sociocultural (Moreno y Pol, 2000; Blanco, 1985; Carpio, 2000; Arenas 2003, los de bienestar físico, mental y social en tanto ajuste o coincidencia entre situaciones objetivas y subjetivas (Levy y Anderson 1980; Rubina y Rebaza 2000; Carpio 2000) las condiciones que permiten a los individuos realizarse de acuerdo con valores ideológicos establecidos por su grupo social (encuesta de CV en España); como representación social de actitudes, imágenes y valores que el colectivo comparte (Moreno y Pol 2000); percepción personal de la situación de vida (Aron, 2002) y aquellos que destacan lo psicológico (Márquez, 2000); lo psicosocial (Tonon, 2004; Laviano, 2004) e incluso lo espiritual (Grimaldo, 2003).

Para Ardila (2003) la calidad de vida es un estado de satisfacción general derivado de la realización de las potencialidades de la persona. Posee aspectos subjetivos y aspectos objetivos. Es una sensación subjetiva de bienestarfísico, psicológicoy social. Incluye como aspectos subjetivos la intimidad, la expresión emocional, la seguridad percibida, la productividad personal y la salud percibida. Como aspectos objetivos el bienestar material, la relaciones armónicas con el ambiente físico y social y con la comunidad, y la salud objetivamente percibida". Las características de la CV (Wikipedia 2007) están centrados en lo siguiente: es un concepto subjetivo, universal, holístico, dinámico y de interdependencia.

En el Manual diagnóstico y estadístico de los trastornos - DSM IV, los trastornos de ansiedad son agrupados en diversos grupos, entre los que se incluye al trastorno de ansiedad generalizada.

\section{Trastorno de ansiedad generalizada}

El TAG estuvo mal definido en el DSM III (APA, 1980), se le conceptualizó como una entidad propia e independiente de los síndromes de ansiedad, cuya sintomatología primaria era la "expectativa de aprensión" o preocupación bastante más específico con múltiples aspectos de la vida. En esta revisión, al diagnóstico del TAG se incluyen los síntomas centrales de "ansiedad y preocupación no realistas o excesivas"; sin embargo, la propuesta del DSM-IV mejora notoriamente los criterios para el diagnóstico de este trastorno.

Las características diagnósticas señaladas por el DSMIV se fundamentan en los siguientes criterios:

a) La ansiedad y la preocupación excesiva (expectación aprensiva) que se observa durante un periodo superior a los 6 meses y que se centran en una amplia gama de acontecimientos.

b) El individuo tiene dificultades para controlar este estado de constante preocupación.

c) La ansiedad y la preocupación se acompañan de al menos otros tres síntomas: inquietud, fatiga precoz, dificultades para concentrase, irritabilidad, tensión muscular y trastornos del sueño.

d) Las situaciones que originan ansiedad y preocupación no se limitan a las que son propias de otros trastornos.

e) Aunque los individuos con TAG no siempre reconocen que sus preocupaciones resultan excesivas, manifiestan una evidente dificultad para controlarlas y les provocan malestar subjetivo o deterioro social, laboral. 
f) Esta alteración no obedece a efectos fisiológicos directos de una sustancia o una enfermedad médica, un trastorno psicótico o un trastorno generalizado del desarrollo.

\section{MATERIAL Y MÉTODOS}

Se empleó el método descriptivo correlacional y como diseño metodológico el descriptivo correlacional comparativo.

\subsection{Población y muestra}

Del universo de tres grupos representativos de pobladores de un sector urbano, marginal y rural del distrito de Huancayo, se tomó una muestra por juicio de 364 sujetos; varones (159) y mujeres (205), de 35 años de edad promedio, pertenecientes a diferentes condiciones socioeconómicas de vida, nivel educativo y otras variables sociodemográficas. De la zona urbana (116), de la zona marginal (133) y de la zona rural (115). Son pertenecientes a Batanyacu (marginal), Parra (urbano) y Aza (rural) de Huancayo.

Los pobladores de las tres zonas de Huancayo se dedican, el $32 \%$ como amas de casa (mujeres), $15 \%$ comerciantes, $12 \%$ estudiantes y el $10 \%$ agricultores (varones), en el nivel de estudios, el $38 \%$ tienen secundaria completa, el $26 \%$ educación superior y el $22 \%$ educación primaria, sólo el $2 \%$ son analfabetos.

En su situación laboral, el $55 \%$ son independientes y el $30 \%$ contratados $y$, cuyos ingresos en la mayoría de los casos (64\%), fluctúan entre S/. 499 a100, el $19 \%$ tienen ingresos entre S/. 990 a 500 y sólo el $6 \%$, más de $\mathrm{S} / 1000.00$ Sólo el $18 \%$ goza vacaciones y el $82 \%$ no; el $59 \%$ tiene más de 40 horas, semanales de trabajo y el $31 \%$ menos de 40 horas y el $75 \%$ trabajan sábados y el $45 \%$ domingos y el $55 \%$ no lo hacen.

\subsection{Técnica, instrumentos y procedimientos de recolección de datos}

Para la recopilación de datos se utilizó dos instrumentos:

- Escala de calidad de vida (ECV) en la versión de Olson y Barnes (Grimaldo, 2003). Un instrumento de 24 ítems, con respuestas según la gradación de Lickert, mide el grado de satisfacción con siete dimensiones de calidad de vida:

1. Hogar y bienestar económico.

2. Amigos, vecindario y comunidad.

3. Vida familiar y familia extensa.

4. Educación y ocio.

5. Medios de comunicación.

6. Religión.

7. Salud.

La ECV según Olson y Barnes (1982 cit. por Grimaldo, 2003) presenta una confiabilidad de .64; por su parte, Grimaldo en Lima obtuvo .86 para la muestra total (.83 para colegios particulares y .86 para colegios estatales), datos que son indicadores positivos precisos para evaluar la calidad de vida. (Cf. Anexo A).

- Cuestionario de preocupación Pensilvania State (PSOW) en la versión de Meyer, Meteggar y Berkovec (Cf. Sandín \& Chorot, 2002) mide el trastorno de ansiedad generalizada (TAG).

Un instrumento de 16 ítems con 5 alternativas de respuestas según la gradación de Lickert. Es un buen instrumento para diferenciar pacientes con TAG de otros trastornos de ansiedad, así como de la población clínica. Una versión española empleada por ellos, se usa en este estudio. (Cf. Anexo B).

\subsection{Procedimiento}

Para la selección del grupo representativo se utilizó la técnica estadística probabilística para determinar el tamaño de la muestra. Los dos instrumentos fueron aplicados de manera individual. Los datos obtenidos, se calificaron e ingresaron a una base de datos para ser sometidos a tratamiento estadístico inferencial por medio de la Prueba de Chi cuadrada al 0,05 y 0,01 de significación estadística.

\section{RESULTADOS}

Los datos obtenidos en cada uno de las tablas se expresan en resultados porcentuales y chi cuadrada al $0,5\left(^{*}\right)$ y $0,1\left(^{* *}\right)$ de significación estadística. 


\subsection{Trastorno de ansiedad generalizada y calidad de vida}

La Tabla 4 indica la relación de las variables calidad de vida (CV) y trastorno de ansiedad generalizada (TAG). Las siglas de las 4 columnas de CV, indican: $\mathrm{MCV}=$ mala calidad de vida, TBCV = Tendencia a baja calidad de vida, TCVB = Tendencia a calidad de vida buena y CVO = calidad de vida optima.

En esta tabla se advierte relación asociativa entre trastorno de ansiedad generalizada y calidad de vida. La mayoría de los pobladores presentan mala calidad de vida sin consideración de si tienen trastorno de ansiedad generaliza o no, pues el $37 \%$ muestra ausencia de TAG y el $34 \%$ si presenta TAG. Los sujetos sin trastorno de ansiedad generalizada tienen tendencia a baja calidad de vida (12\%) y sólo el $6 \%$ tendencia a calidad de vida buena. Lo pertinente en estos resultados es la existencia de diferencias de los sujetos en sus niveles de CV y que ellos teniendo puntuaciones elevadas de baja CV, también pueden presentar trastornos de ansiedad generalizada. Se concluye que, existe una tendencia a mejores indicadores de CV y ausencia de TAG.

Tabla 4. Trastorno de ansiedad generalizada y calidad de vida.

\begin{tabular}{|c|c|c|c|c|c|c|c|c|c|c|}
\hline \multirow[t]{2}{*}{ TAG } & \multirow[t]{2}{*}{ CV } & \multicolumn{2}{|c|}{$\begin{array}{c}\text { MCV } \\
n=259\end{array}$} & \multicolumn{2}{|c|}{$\begin{array}{c}\text { TBCV } \\
\mathrm{n}=77\end{array}$} & \multicolumn{2}{|c|}{$\begin{array}{l}\text { TCVB } \\
\mathrm{n}=26\end{array}$} & \multicolumn{2}{|c|}{$\begin{array}{l}\text { CVO } \\
n=2\end{array}$} & \multirow[t]{2}{*}{$X^{2}$} \\
\hline & & & $\%$ & $f$ & $\%$ & & $\%$ & $f$ & $\%$ & \\
\hline SINTAG & & 135 & 37 & 45 & 12 & 21 & 6 & 1 & 0 & * \\
\hline CONTAG & & 124 & 34 & 32 & 9 & 5 & 1 & 1 & 0 & \\
\hline
\end{tabular}

\subsection{Trastorno de ansiedad generalizada y calidad de vida en razón del género}

La tabla 5 muestra la relación de estas dos variables con el género de los sujetos.

Tabla 5. Trastorno de ansiedad generalizada y calidad de vida en razón del género.

\begin{tabular}{|c|c|c|c|c|c|c|c|c|c|c|}
\hline \multirow[t]{2}{*}{ TAG } & \multirow[t]{2}{*}{$\mathrm{CV}$} & \multicolumn{2}{|c|}{ MCV } & \multicolumn{2}{|c|}{ TBCV } & \multicolumn{2}{|c|}{ TCVB } & \multicolumn{2}{|c|}{ cVo } & \multirow{2}{*}{$x^{2}$} \\
\hline & & $M$ & $\mathrm{~V}$ & $M$ & $\mathrm{~V}$ & $M$ & V & $M$ & V & \\
\hline SIN TAG & & 77 & 58 & 17 & 28 & 13 & 8 & 1 & 0 & \\
\hline CON TAG & & 79 & 45 & 15 & 17 & 3 & 2 & 0 & 1 & ns \\
\hline
\end{tabular}

$P<0,05=n s$

Aquí es notable la ausencia de significación estadística en razón del género, los resultados evidencian puntuaciones similares en varones y mujeres. La dirección es similar al cuadro precedente en los que mayor proporción de sujetos, tanto varones y mujeres, acusan mala calidad de vida y tendencia a baja calidad de vida.

\subsection{Trastorno de ansiedad generalizada y calidad de vida según la zona}

En la Tabla 6 se advierte los resultados de la comparación de las variables TAG y CV en razón de las zonas: urbana, marginal y rural.

Tabla 6. Trastorno de ansiedad generalizada y calidad de vida en razón de la zona.

\begin{tabular}{|c|c|c|c|c|c|c|c|c|c|c|c|c|c|}
\hline \multirow[b]{2}{*}{ TAG } & \multicolumn{3}{|c|}{ MCV } & \multicolumn{3}{|c|}{ TBCV } & \multicolumn{3}{|c|}{ TCVB } & \multicolumn{3}{|c|}{ CVO } & \multirow[b]{2}{*}{$X^{2}$} \\
\hline & U-M & $U$ & $\mathrm{R}$ & U-M & $U$ & $\mathrm{R}$ & U-M & U & $\mathrm{R}$ & U-M & U & $\mathrm{R}$ & \\
\hline SIN TAG & 32 & 34 & 69 & 31 & 8 & 6 & 13 & 7 & 1 & 1 & 1 & 0 & \\
\hline CON TAG & 35 & 57 & 32 & 16 & 9 & 7 & 4 & 1 & 0 & 1 & 0 & 0 & ns \\
\hline
\end{tabular}

$n s=p>0,05$ 
Esta variable sociodemográfica no guarda asociación significativa con las variables CV y TAG. Los sujetos de las zonas: urbana, marginal y rural no presentan diferencias, es notable que la mayoría de los pobladores de las tres zonas muestren mala calidad de vida, pero con una ligera variación aunque estadísticamente no significativa, los sujetos de las zonas: urbana y urbano- marginal tiene puntuaciones algo altas de TAG y los de zona rural (mayor proporción de ellos), no presenta trastornos de ansiedad generalizada y, solamente los sujetos de la zona marginal con tendencia a calidad de vida buena muestran ausencia de TAG.

\subsection{Trastorno de ansiedad generalizada y dimensiones de calidad de vida}

En la tabla 7 se observa la relación del trastorno de ansiedad generalizada con cada una de las dimensiones de la calidad de vida, Con TAG (CT), Sin TAG (ST): Hogar y bienestar económico (H y BE), Amigos, vecinos y comunidad ( $A, V$ y $C$ ), Vida familiar y familia extensa (VF y FE), Educación y ocio (E y O), Medios de comunicación (MC), Religión (R) y Salud (S). La numeración debajo de cada dimensión indica: 1 (CVO), 2 (TCVB), 3 (TBCV) y 4 (MCV).

Tabla 7. Trastorno de ansiedad generalizada e indicadores de calidad de vida

\begin{tabular}{|c|c|c|c|c|c|c|}
\hline $\begin{array}{c}\mathrm{H} \text { y } \mathrm{BE} \\
\mathrm{X}^{2}\end{array}$ & $\begin{array}{c}A, V, C \\
X^{2}\end{array}$ & $\begin{array}{c}\text { VF y FE } \\
X^{2}\end{array}$ & $\begin{array}{l}E-O \\
X^{2}\end{array}$ & $\begin{array}{c}M C \\
X^{2}\end{array}$ & $\begin{array}{l}\mathrm{R} \\
\mathrm{X}^{2}\end{array}$ & $\begin{array}{l}S \\
X^{2}\end{array}$ \\
\hline 1234 & 1234 & $\begin{array}{llll}1 & 2 & 3 & 4 \\
\end{array}$ & 12234 & 1234 & $\begin{array}{l}1234 \\
\end{array}$ & 1234 \\
\hline S T 75405135 & 116552116 & 183232123 & 77743617 & 54496536 & 27617145 & 48635231 \\
\hline CT 70353618 & $11126121^{\text {** }}$ & 101729103 & $94431211^{\text {** }}$ & 42367012 * & 16356643 & 41472636 \\
\hline
\end{tabular}

Se observa que las puntuaciones más altas ocurren en las dimensiones: amigos, vecinos - comunidad, educación - ocio y medios de comunicación, posiblemente por que le asignan mayor relevancia y, en estos casos se vincula con ausencia de trastorno de ansiedad generalizada.

Estas dimensiones les producen mayor satisfacción, siendo más acentuada en el sector rural donde también la religión y la salud destacan en comparación con los otros sectores; para la zona marginal, la vida familiar y la vida extensa tienen cierta relevancia en su CV. En general, es posible sostener que los indicadores de carácter social, el ocio, la educación y los medios de comunicación tiene más significado en la calidad de vida de los pobladores del Valle del Mantaro, casi independiente del papel que puede representar el trastorno de ansiedad generalizada en tanto dimensión psicológica de desajuste; por eso incluso en el área de la salud aparece con baja puntuación.

\section{DISCUSIÓN}

Sin duda, los hallazgos de esta investigación revelan en los pobladores del Valle del Mantaro la existencia de bajos niveles de calidad de vida asociados posiblemente con las condiciones socioculturales de existencia que socializan en ellos, percepciones de insatisfacción, en especial en los dominios interpersonales (amigos, vecinos, comunidad), educación, ocio y medios de comunicación, situación preocupante que demanda el diseño de programas de promoción e intervención que pueda revertir dicho problema. Esta variable, por su naturaleza compleja y multidimensional, se asocia con el trastorno de ansiedad generalizada de manera directa e inversa, pues un porcentaje elevado no presenta TAG contra otro que acusa en los niveles clínicos, por eso personas con mala calidad de vida y con indicadores de trastorno de ansiedad generalizada difícilmente podrán enfrentar los problemas que les aqueja en su vida personal y social.

Estudios de la OMS indican que la prevalencia de TAG llega al $5 \%$ y en centros médicos al $12 \%$; a nivel nacional. En el Valle del Mantaro un problema de ribetes clínicos que dicho trastorno alcanza el $34 \%$.

Puede pensarse que por problemas socioculturales de la población se esté generando un estilo de vida con características particulares que configuran una subcultura donde los pobladores aprenden a 
vivir, a limitar sus aspiraciones, a no sucumbir, pero mantenerse resilientes y conformistas. Alarcón (1986) manifiesta que pueden ser alteraciones del comportamiento humano por efectos de la pobreza.

Las variables sociodemográficas como el género y el tipo de zona no marcan diferencias en la calidad de vida ni en el trastorno de ansiedad en pobladores andinos, existe un proceso de homogenización y ante los cuales se requiere otro tipo de mirada en campo de las ciencias sociales, en especial a la psicología social, es mucho que le queda por hacer en este terreno, tanto desde el punto de vista teórico-conceptual como desde punto de vista práctico aplicado.

Compartimos con Tonon (2004) que se requiere nuevas actitudes profesionales tendentes a un trabajo desde las potencialidades que desde las carencias y con un anclaje comunitario de tipo psicosocial que incluye no solo el análisis sociopolítico sino responder con programas y evaluaciones de intervenciones psicosociales como tarea interdisciplinar necesaria y urgente, para buscar el cambio social para buscar el cambio personal.

De acuerdo con el problema, se requiere una educación liberadora orientada a la calidad de vida, que finalmente hará posible que la generación presente de los pobladores de Huancayo y varios grupos humanos del país, puedan revertir la cultura de la pobreza, la marginación, el conformismo y la mala calidad de vida.

\section{CONCLUSIONES}

- Se confirma la hipótesis, existe relación significativa entre calidad de vida y trastorno de ansiedad generalizada; la mayoría de pobladores del valle del Mantaro, presenta baja calidad de vida e indistintamente presencia y ausencia de trastorno de ansiedad generalizada.

- Las variables sociodemográficas, género y tipo de zona, no se asocian significativamente con calidad de vida y trastorno de ansiedad generalizada; los pobladores del Valle del Mantaro no difieren en razón del género y la zona (urbana, urbano-marginal y rural).
- Las dimensiones: amigos, vecinos-comunidad, educación-ocio y medios de comunicación de la calidad de vida, tienen mayor significado en los pobladores de Huancayo aun cuando presenten puntuaciones bajas.

- Así, es posible sugerir: la necesidad de diseñar programas de promoción para mejorar la calidad de vida y estrategias de prevención para reducir la presencia de trastornos de ansiedad generalizada en la población del Valle del Mantaro y la realización de investigaciones sobre psicología de la salud y calidad de vida en niños y adolescentes.

\section{LITERATURA CITADA}

Alarcón, R. 2000. Psicología de la pobreza. En: R. Alarcón. Psicología, pobreza y subdesarrollo. Lima: INIDE; Cap. 3, 85-122.

Alarcón, R. 2001. Variables psicológicas y sociodemográficas asociadas con la felicidad. Memorias. X Congreso Nacional de Psicología. Lima: Colegio de Psicólogos del Perú, 1;set., 12-15.

Ardila, R. 2003. Calidad de vida: una definición integradora. Revista Latinoamericana de Psicología. 35 (2): 161-164. (fecha de acceso 5 de octubre de 2006) disponible en: psychology@ latino.net.co.

Arenas, C. 2003. Psicología comunitaria y calidad de vida en el Perú de contrastes. Revista de Psicología (UNMSM) ; VI (2): 99-110.

Barbarán, T., Flores, E., Vásquez, J. y Tomás A. 2003. Calidad de vida y soporte social en víctimas del terrorismo. Paradigmas, Revista psicológica de actualización profesional. 4 (6): 49-58.

Blanco, A. 1991. La calidad ambiental: supuestos psicosociales. J. F. Morales. Psicología social aplicada. Bilbao (España): Desclée de Brouwer, 159-182.

Brannon, J. F. y Feist, J. 2001. Psicología de la salud. Madrid: Paraninfo. 
Cornejo, W. y Pérez, B. 1999. Satisfacción residencial y actitud ambiental en conjuntos habitacionales de Huancayo. Informe final de investigación. Huancayo: Facultad de Pedagogía y Humanidades, Universidad Nacional del Centro del Perú.

Cornejo, W. y Cornejo, G. 2002. Indicadores psicosociales y ambientales de calidad de vida, síndrome de burnout y motivación de docentes de lca. Educación ambiental. III Congreso Nacional de Educación ambiental. 2-4 agosto. Universidad Marcelino Champagnat, Centro de Proyección Social; 84.

Domínguez, B. y Cols. 2001. Calidad de vida. Manual para la evaluación y control del estrés. Lima: Cuadernos Psicológicos-CEDEIS. Temas monográficos de Psicología; № 4.

DSM-IV. 1995. Manual diagnóstico y estadístico de los trastornos mentales. Barcelona: Masson, S.A.

Grimaldo, M.P. 2003. Escala de calidad de vida de Olson y Barnes. Manual técnico. Lima: USMP. Escuela de Psicología, Instituto de Investigación.

Guerra, L.F. Calidad de vida y autoestima en adolescentes de la institución educativa San Ramón - Cajamarca. (Tesis Licenciatura). Facultad de Psicología, Universidad Privada Antonio Guillermo Urrelo. Cajamarca.

Inga, J. 1999. Interacciones entre niveles de ansiedad y de autoestima en un grupo de estudiantes de una Universidad de Lima. Memorias. IX Congreso Nacional de Psicología. Consejo Directivo Nacional. Colegio de Psicólogos del Perú. Lima; 148.

Keller, S.; Williams L.K.; White, V. F. y Alberti, G. 1967 Cambios culturales y estrés en el Perú rural. Informe Preliminar. Lima: IEP.

Lamas, H. y Murrugarra A. 2007. Calidad de vida y salud. Lima: Cuadernos de divulgación, C.Ps.P., C.D.R-I.

Levi, L. y Anderson, L. 1990. La tensión psicosocial. Población, ambiente y calidad de vida. México: El Manual Moderno, S.A.
Maciaaton, D. y Xavier, F. 1999. Líneas actuales de investigación en psicología de la salud. En M.A. Simón. Manual de psicología de la salud. Fundamentos, metodología y aplicaciones. Madrid: Biblioteca Nueva; Cáp. VII, 217-258.

Maric, L. 2005. Indicadores psicosociales de la calidad de vida en la ciudad de El Alto. I Congreso Internacional de Psicología andina. Diagnóstico y perspectivas. Huancayo: UPLA. Resúmenes; sept. (CD ROM).

Márquez, Mạ de la Luz. 2001. Evaluación en calidad de vida. Calidad de vida. VII Seminario Internacional. Lima: Facultad de Ciencias de la Comunicación, Escuela Profesional de Psicología, Universidad San Martín de Porres; 49-78.

Moscoso, M. 1998. Psicología de la salud: Hacia una nueva ciencia de la conducta. Revista de la Facultad de Psicología de la UNMSM; || (1): 107-124.

Oblitas, L.A. Coord. 2005. Psicología de la salud y calidad de vida. México: Thomson.

Oblitas, L.A. Coord. 2005. Psicología de la salud y calidad de vida. México: Thomson.

Otero, H. 1998. Calidad de vida y enfermedad. Revista de la Facultad de Psicología de la UNMSM; II (1): 125-134.

Romero, J. 2001. Calidad de vida. VII Seminario Internacional. Lima: Facultad de Ciencias de la Comunicación, Turismo y Psicología, Escuela Profesional de Psicología, USMP.

Romero, J. 2001. Calidad de vida. VII Seminario Internacional. Lima: Facultad de Ciencias de la Comunicación, Turismo y Psicología, Escuela Profesional de Psicología, USMP.

Rubina, A. 2005. Rebaza E. Bienestar subjetivo en adultos mayores institucionalizados y no institucionalizados. Revista de Psicología (UCSM, Arequipa). 2 (2): 63-77. 
Sandín, B. y Chorot, P. 2002. Síndromes clínicos de la ansiedad. En A. Belloch B.; Sandía \& F. Ramos. Manual de psicopatología. Vol. 2. Madrid: McGraw-Hill; Cap. 3, pp. 81-112.

Tonon, G. 1993. Calidad de vida y desgaste profesional. Una mirada del síndrome de burnout. Buenos Aires: Espacio.
Wikipedia, 2007. Calidad de vida. (Fecha de acceso el 3 de enero de 2007) disponible en: http://es. wikipedia.org/wiki/calidad_de_vida.

Zinmermann, M. 1998. Psicología ambiental y calidad de vida. Bogotá: ECOE. 